\title{
Ontologies in System Engineering: a Field Report
}

\author{
Marco Menapace and Armando Tacchella \\ DIBRIS - Università degli Studi di Genova - Viale F. Causa 13, 16145 Genova \\ marco.menapacededu.unige.it-armando.tacchella@unige.it
}

September 18, 2018

\begin{abstract}
In recent years ontologies enjoyed a growing popularity outside specialized AI communities. System engineering is no exception to this trend, with ontologies being proposed as a basis for several tasks in complex industrial implements, including system design, monitoring and diagnosis. In this paper, we consider four different contributions to system engineering wherein ontologies are instrumental to provide enhancements over traditional ad-hoc techniques. For each application, we briefly report the methodologies, the tools and the results obtained with the goal to provide an assessment of merits and limits of ontologies in such domains.
\end{abstract}

\section{Introduction}

Ontologies are witnessing an increasing popularity outside specialized AI communities. While this is mostly due to Semantic Web applications [1], we must also credit their ability to cope with taxonomies and part-whole relationships, to handle heterogeneous attributes, and their provision for various automated reasoning services - see, e.g., [2]. These features have been recognized since long time in system engineering, the community encompassing all areas of research devoted to design, implementation, monitoring and diagnosis of technical processes. For instance, in the operations and maintenance sub-community, the use of ontologies is explicitly advocated 11 Also standards like ISO 13374 (Condition monitoring and diagnostics of machines - Data processing, communication and presentation) suggest the use of ontologies for several tasks, mostly related to data conceptualization. However, the adoption of ontologies faces some challenges, mostly due to speed and reliability constraints imposed by industrial settings.

Here we investigate this issue by considering four contributions of ours to application domains wherein ontologies provide key capabilities in system engineering. The first case study is about an on-board rolling-stock condition analyzer, i.e., a system to perform fault detection and classification [3]. The second one is about monitoring an intermodal logistic system [4]. The third one is about an ontology-based framework to generate diagnostic-decision support systems [5]. Finally, a fourth case study is an

\footnotetext{
${ }^{1}$ See, e.g., the MIMOSA open standard architecture at www . mimosa . org
} 
application to computer-automated design of elevator systems. In the following, we briefly introduce each case study, giving details about its context, underlying motivation and intended objectives. The ultimate goal of the paper is to discuss and compare the results obtained to assess the effectiveness of ontologies in such application domains.

Ontologies for condition analysis. We introduced an ontology-based condition analyzer (CA) [3] in the context of the EU project Integrail2. Our CA collects signals from control logic installed on locomotives, and it leverages an ontology to correlate observed data, symptoms and faults. The CA must mate two competing needs: $(i)$ railway regulations require hardware which is highly reliable, and whose performances are thus far even from desktop workstations; ( $i i)$ ontology-related tools, e.g., description logic reasoners, have relatively large memory, processor and storage footprints. In this experience, the main goal was thus to check whether reasoning with ontologies can provide useful diagnostic feedback in a resource-restricted scenario.

Ontologies for system monitoring. In [4] we provided strong evidence of practical uses for ontologies in complex systems engineering by implementing a monitor for Intermodal Logistics Systems (ILSs), i.e., systems supporting the movement of containerized goods. In particular, we considered combination of rail and road transport, where rail transport is provided by short-distance shuttle trains, and network coverage is achieved through connections at specialized terminals. In this experience, the main goal was to gather data about terminal operations and compute global performances indicators, where access to data is mediated by an ontology — ontology-based data access (OBDA) [6]. Here, unlike the CA case study, the ability to handle large amount of data is crucial, but reasoning is limited to SPARQL query answering.

Ontologies for diagnostic support system generation. Diagnostic Decision Support Systems (DDSSs) help humans to infer the health status of physical systems. In [5] we introduced DiSeGnO - for "Diagnostic Server Generation through Ontology" - to generate customized DDSSs. As in the ILS monitoring case study, since it is expected that large quantities of data should be handled, the ontology language is restricted to those designed for tractable reasoning — see, e.g., [6]. In this case, ontology-based reasoning is not leveraged, as $\mathrm{DiSeGnO}$ generates relational databases from the domain ontology and then computes diagnostic rules with PTOLEMY II [7], an open-source software simulating actor-based models.

Ontologies for computer-automated design. As mentioned in [8], the first scientific report of intelligent computer-automated design (CautoD) is the paper by Kamentsky and Liu [9], who created a computer program for designing character-recognition logic circuits satisfying given hardware constraints. In mechanical design — see, e.g., [10] — the term usually refers to techniques that mitigate the effort in exploring alternative solutions for structural implements. In our LIFTCREATE CautoD program for elevator systems 3 , ontologies support intelligent design creation and optimization by managing detailed part-whole taxonomies, wherein different relations among components can be expressed. This case study provides thus yet another application of ontologies, mostly oriented to intelligent computation and data persistency.

\footnotetext{
${ }^{2}$ More details about Integrail at http://www. integrail.eu/

${ }^{3}$ Part of the AILIFT software suite www . ailift.it
} 


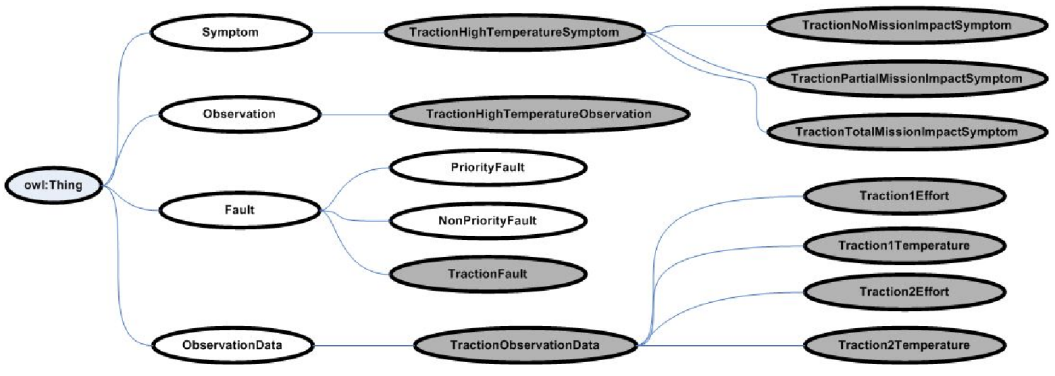

Figure 1: A portion of the E414 ontology regarding traction faults. Concepts are nodes and object properties are edges: white nodes are SP3A concepts, grey ones are E414specific concepts.

Overall, the case studies considered witness the great flexibility that ontologies provide in handling diverse application scenarios, from condition analysis of locomotives, to automated design of elevators, considering both cases wherein they provide the basis for logic reasoning services, or just advanced data-modeling capabilities. The rest of the paper is structured as follows. In Sections 2, 3, 4 and 5 we sketch the design, the implementation and the results obtained in the case studies described above. Section 6 concludes the paper by summarizing the results and providing some discussion thereof.

\section{Rolling stock condition analysis}

The CA prototype described in [3] focuses on fault detection on Trenitalia E414 locomotive. The main task of the $\mathrm{CA}$ is to perform fault classification according to priority for maintenance, and impact on mission-related and safety-related aspects. Here, we focus on traction groups as an example of subsystem that can generate a faulty condition. Our ontology for the E414 locomotive is written in OWL 2 language and it builds on the SP3A core ontology - see [3] for details. In particular, the E414 ontology leverages the SP3A concepts of OBSERVATIONDATA, i.e., process variables, and OBSERVATION, i.e., sequences of observation data from which individuals of class SYMPTOM and FAULT arise. SYMPTOM individuals are related to OBSERVATION individuals via the REFERSTOOBSERVATION property and to FAULT individuals via the REFERSTOFAULT property. FAULT is a concept whose individuals are defined in terms of the necessary HASSYMPTOM relationship with SYMPTOM individuals. Two subclasses of FAULT are defined: PriorityFAULT and NONPRIORITYFAULT, with obvious meaning. In Figure 1 we show a portion of the E414 ontology related to traction faults, where concepts have been specialized in subclasses whose individuals correspond to actual signals and subsystems. Fault and symptom classification is obtained by a Description Logic (DL) reasoner considering the patterns observed. For instance, in the case of TRACTIONHIGHTEMPERATUREOBSERVATION, three ranges of temperatures are defined that correspond to "interesting" patterns: from 70 to 80 degrees, from 80 to 130 degrees, and over 130 degrees. It is postulated that observations falling in the 
Table 1: Results with (a) lazy and (b) eager implementations of the CA.

\begin{tabular}{|c|c|c|c|}
\hline Scenario & Memory Consumption [MB] & CPU Time [ms] & Amortized CPU Time [ms] \\
\hline 1a & 38 & 90 & ND \\
2a & 74 & 25373 & 25373 \\
3a & 106 & 1053656 & 210731 \\
4a & OUT OF MEMORY & 3253637 & 191390 \\
\hline 1b & 37 & 90 & ND \\
2b & 72 & 21506 & 21506 \\
3b & 104 & 86938 & 17387 \\
4b & 105 & 279523 & 16442 \\
\hline
\end{tabular}

second and in the third ranges are to be considered mission critical, while the ones in the first category are only maintenance critical.

A detailed description of the CA architecture can be found in [3]. Here we provide some intuition on how the analyzer works considering high temperatures in the traction groups. When the temperature of a group is higher than 70 degrees for at least 3 consecutive samples read from the field bus, the CA starts tracking a potential anomalous pattern. Once such a pattern is detected, the corresponding individuals in the classes TRACTIONOBSERVATIONDATA and TRACTIONHIGHTEMPERATUREOBSERVATION are recorded. SYMPTOM individuals are built along with all the properties required by the ontology specification. For example, if an observation of the class TRACTIONHIGHTEMPERATUREOBSERVATION has been created, a specific individual TRACTIONHIGHTEMPERATUREOBSERVATION is related to a new SYMPTOM individual by the REFERSTOOBSERVATION property. FAULT individuals for each SYMPTOM individual are created together with the CAUSEDBYSYMPTOM property. FAULT as well as SYмРтом individuals are built of generic type, leaving their classification to the DL reasoner. Once the classification is done, the CA publishes the results, transmitting them to external agents. As an example, let us assume that $i$ is an individual of the class TRACTIONHIGHTEMPERATUREOBSERVATION whose property ISAT is set to the constant_130DEGREES, $s$ is the SYMPTOM individual related to $i$, and $f$ is the FAULT individual related to $s$. The E414 ontology postulates that all symptoms such that the corresponding observation is an instance of TRACTIONHIGHTEMPERATUREOBSERVATION related by ISAT to the constant _130DEGREES are also an instance of TRACTIONTOTALMISSIONIMPACTSYMPTOM, which is a subclass of SYMPTOM. Therefore, a reasoner can infer that $s$ belongs to MisSIONRELATEDSYMPTOM.

Out of the three sets of experiments performed in [3], we report just those to ensure that the CA implementation fits the constraints. To this end, we ran several tests using different fault scenario 4 . Table 1 shows the results obtained by running the CA on four different scenarios - the first includes no fault, the second includes only one fault, the third includes five contemporary faults, and the last 17 contemporary faults — using two different configurations. Configuration (a) is "lazy", i.e., it keeps all the individuals, while configuration (b) is "eager", i.e., it deletes individuals as soon as possible. As we can see in Table 1, the eager version results in a great improvement

\footnotetext{
${ }^{4}$ Sets of multidimensional time series $(3600$ samples at $1 \mathrm{~Hz}$ ) corresponding to 52 process variables are generated. Simulations run on EN50155-compliant embedded devices with $1 \mathrm{GHz}$ Socket 370 FC-PGA Celeron Processor with 256MB of main memory and a 1GB SSD running Linux Blue Cat (kernel 2.6) and Sun Java Virtual Machine implementation (JRE 1.6). The DL reasoner is PELLET [11].
} 


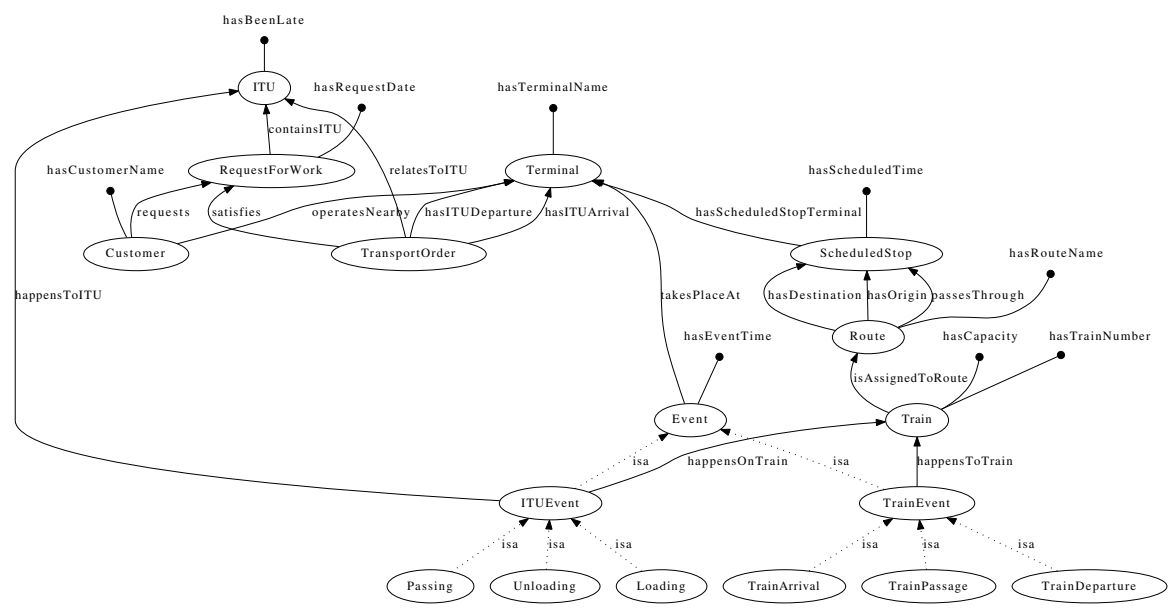

Figure 2: ILS ontology describing the design of the OBDA solution. Ellipses denote concepts with datatype properties; directed edges are object properties; dotted edges are concept inclusions.

over the lazy one, both in terms of memory consumption and in terms of computation time. In particular, in the second column of Table 1 we can notice that the eager version performs reasonably well, even in the fourth test case (worst-case scenario). In the same scenario, the lazy version exceeds the amount of available memory. As we can see in the rightmost column of Table 1 , the amortized computation time over a single scenario decreases with the number of concurrent observations detected in the round. Managing a round of samples without detected observations takes only $90 \mathrm{~ms}$, which leaves enough time for other activities, and allows the CA to process all the incoming signals in due course.

\section{Monitoring of intermodal systems}

In [4] we provided evidence that ontology-based data access (OBDA) [6] is of practical use in the context of Intermodal Logistics Systems (ILSs). The investigation focuses on the opportunity to build a monitoring information system (MIS) using OBDA instead of relational databases (RDBs). The application scenario is an ILS relying on a logic akin to computer networks, i.e., frequent short-distance trains with a fixed composition and a predefined daily schedule to cover some geographical area. Intermodal Transport Units (ITUs) enter the network at some terminal and travel to their destination according to a predefined route, usually boarding more than one train along the way. Terminals collect ITUs from areas of approximately $150 \mathrm{Km}$ in radius in order to minimize road transport. The MIS is a key enabler to minimize delivery time, maximize rolling-stock and network utilization and, ultimately, reduce the economic overhead of transportation for the final customer. The main goal of the MIS is to compute Key Performance Indicators (KPIs) to perform tactical and strategical decision making about 


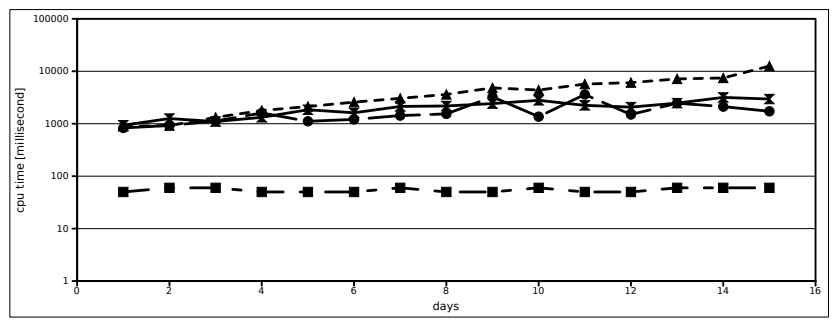

Figure 3: Computation time of a KPI with different query processors: SQL (square), ARQ (circle), PELlET (hourglass), QUEST (triangle). In each plot, the $x$ axis displays the number of simulation days from 1 to 15 , the $y$ axis displays the CPU time (in milliseconds on a logarithmic scale).

the network.

In Figure 2 we present a graphical outline of the ontology at the heart of our OBDA solution to monitor the ILS. The ontology - ILS ontology in the following — is compliant with the OWL 2 QL profile described in the official W3C's recommendation as "[the sub-language of OWL 2] aimed at applications that use very large volumes of instance data, and where query answering is the most important reasoning task.". Given the ILS application domain, OWL 2 QL guarantees that conjunctive query answering and consistency checking can be implemented efficiently with respect to the size of data and ontology, respectively. The restrictions that OWL 2 QL implies did not hamper the modeling accuracy of our ILS ontology. In Figure 2 we can pinpoint classes related to freight forwarding such as Customer, i.e., companies forwarding their goods through the network, RequestForWork, i.e., the main document witnessing that a given customer has issued a request for transporting a number of ITUs, TransportOrder, i.e., the "bill of transit" associated to each ITU, as well as entities related to physical elements such as ITU, Terminal and Train. Also "logical" entities are modeled such as Route, i.e., a sequence of terminals and railway connections serviced regularly by one or more scheduled trains and ScheduledStop, i.e., terminals associated to a given route with a given schedule. Event is the main monitoring entity, as the calculation of most KPIs relies on the exact recording of events at specific locations.

To assess OBDA performances, in [4] we obtained different artificial utilization scenarios by changing the number of ITUs shipped daily from each terminal. Considering typical usage patterns, we postulated that a provision of 10 to 50 ITUs is to be shipped daily from each terminal, with 40 to 50 ITUS corresponding to a heavy utilization. Scenarios are simulated for an increasing number of days to evaluate scalability, and all of them share common settings as far as number of train travels, number of cars per train, and timetabling are concerned. Unexpected delays as well as the number of customers per terminal follow a probabilistic model - see [4] for more details. In Figure 3 we display the result 5 obtained in the case of an heavy utilization scenario

\footnotetext{
${ }^{5}$ All results are obtained on a family of identical Intel-based PCs, featuring a Core2Duo $2.13 \mathrm{GHz}$ CPU, 4GB of RAM and running Ubuntu Linux 10.04 (64 bit edition).
} 


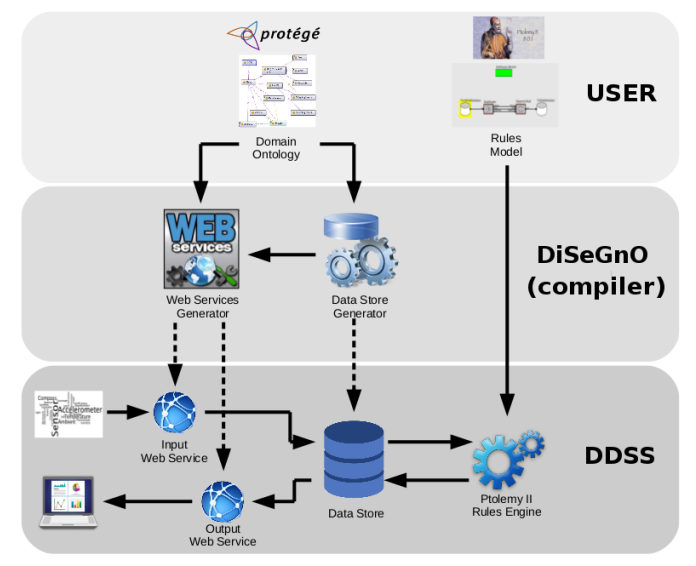

Figure 4: Functional architecture and work-flow of DiSeGnO framework.

to compute a specific KPI, namely the average number of ITUs unloaded per hour. The performance of four different query-answering systems are reported: a SQL query on a native RDB implementation, and a SPARQL query on the ontology store. The SPARQL query can be answered by three different systems, namely ARQ (the default query processor in the JENA library), PELLET (the same DL reasoner that we consider in Section 2] and QUEST [12]. The latter is the only reasoner exploiting the fact that SPARQL queries can be compiled on-the-fly into SQL queries for an equivalent RDB representation of the ontology stored in the main memory. As we can observe in Figure 3, OBDA-based solutions show higher overall computation times than the RDB-based solution - from 1 to 2 orders of magnitude - together with an apparently growing trend associated to the time span of the simulation. However, as we have shown in [4], a trend test performed on the results obtained with the best OBDA solutions for various KPIs, displays no statistically significant increase in the CPU time required to answer various queries with respect to the number of days. Considering that for most KPIs we can adopt an "eager" solution similar to that considered in Section 3 . we can conclude that OBDA is practically feasible for monitoring medium-to-large scale systems.

\section{Diagnostic support systems generation}

In [5] we introduced an approach to compile ontology-based descriptions of equipment into diagnostic decision support systems (DDSSs). The tool DiSeGnO, whose functional architecture and work-flow is sketched in Figure 4, fulfills this task in three phases: in the USER phase, a domain ontology and diagnostic rules model are designed by the user; in the DiSeGnO phase, the system reads and analyzes the ontology and the rules to output the actual DDSS; in the DDSS phase, input web services receive data from the observed physical system and record them in the generated data store. According to the ISO 13374-1 standard a DDSS consists of six modules of which DiS- 


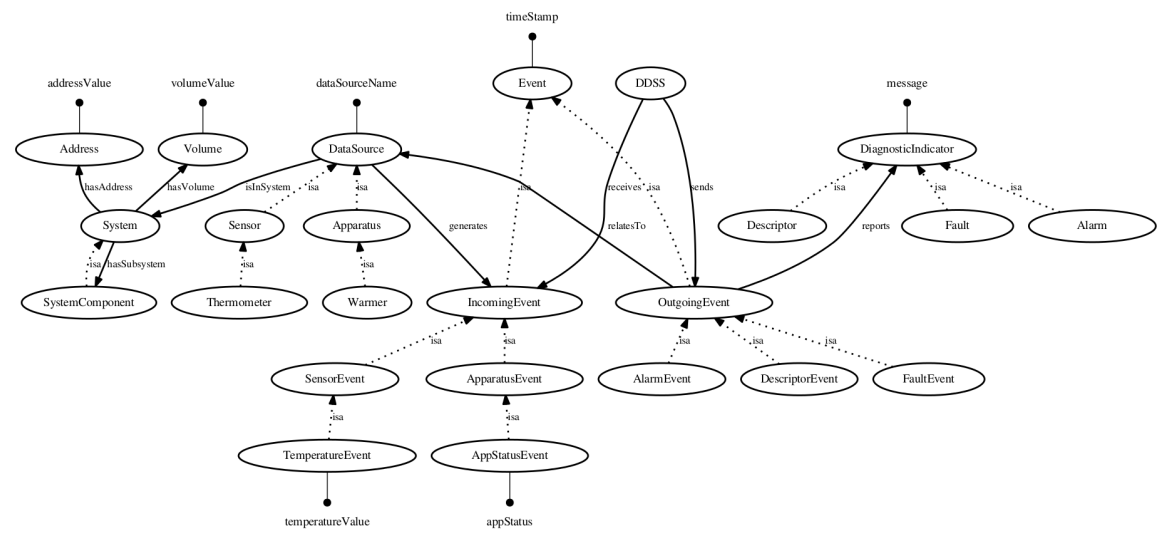

Figure 5: Domain ontology for HVAC monitoring. Formalism is the same as in Figure 2

eGnO implements three: Data Manipulation to perform signal analysis and compute meaningful descriptors, State Detection to check conformity to reference patterns, and Health Assessment to diagnose faults and rate the current health of the equipment or process. As shown in Figure 4 the ontology description is created by a system architect in the USER phase. The ontology must be written using OWL 2 QL language as in the case study shown in Section 3 The diagnostic computation model must be a sound actor diagram generated by PTOLEMY II [7] which describes the processing to be applied to incoming data in order to generated diagnostic events - here we focus on the ontology part, but more details on the rule handling part can be found in [5]. The DiSeGnO phase contains the actual DDSS generation system which consists of the Data Store Generator, i.e., a piece of software that creates a relational database by mapping the domain ontology to suitable tables, and the Web Services Generator, i.e., a module that creates interface services for incoming and outgoing events. Finally, in the DDSS phase, data is acquired and stored in the internal database, the rules engine processes data and generates diagnostic events which are then served to some application.

An example of a DiSeGno-compliant equipment description is shown in Figure 5. The ontology is related to a Heating Ventilation and Air Conditioning (HVAC) appliance and it is divided into a static and a dynamic part. In the static part, which is not updated while monitoring, the ontology contains a description of the observed physical system. In the HVAC ontology we have System and DataSource, related by the isInSystem property. hasSubsystem relationship indicates that one System could be composed by one or more SystemComponent which are themselves subclasses of System. Finally, DataSource is the class of elements that can generate diagnostic-relevant information. The dynamic part describes events, including both the

\footnotetext{
${ }^{6}$ While this can be accomplished in several ways, the tool PROTÉGÉ [13] is suggested because it is robust, easy to use, and it provides, either directly or through plug-ins, several add-ons that facilitate ontology design and testing.
} 


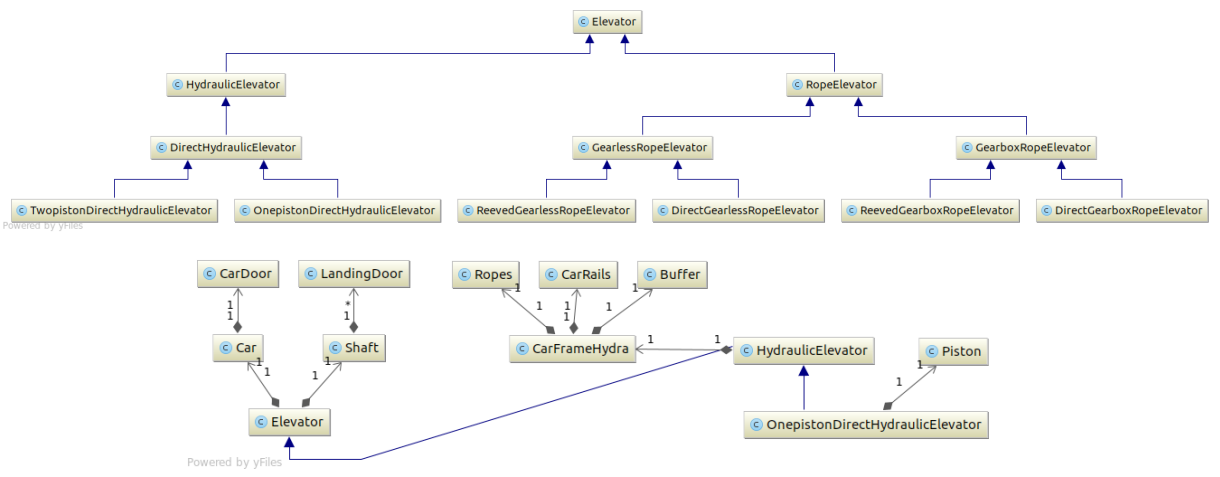

Figure 6: Ontologies describing the implements handled by LIFTCREATE (top) and the components of OnePistonHydraulicElevator (bottom). Concepts are rectangles, concept inclusion is denoted by solid arrows, and HAS-A object properties are denoted by diamond-based arrows.

ones generated by the observed system and its components, and those output by the DDSS. An event is always associated to a time-stamp and it can be either incoming to the DDSS from the observed system, or outgoing from the DDSS7 The main concepts in the dynamic part of the HVAC ontology are DDSS which receives instances of IncomingEvent and sends instances of OutgoingEvent. Notice that IncomingEvent instances are connected to DataSource instances by the role generates, denoting that all incoming events are generated by some data source. Also every OutgoingEvent instance, i.e., every diagnostic event, relatesTo some instance of DataSource, because the end user must be able to reconstruct which data source(s) provided information that caused diagnostic rules to fire a given diagnostic event. OutgoingEvent specializes to AlarmEvent, FaultEvent and DescriptorEvent. Every OutgoingEvent instance is connected to one of DiagnosticIndicator instances, i.e. Alarm, Fault and Descriptor sub-concepts, by reports relation, in order to have a reference message about the diagnostic rules.

\section{Computer-automated design of elevators}

Our latest ontology-based application is in the field of computer-automated design (CautoD) which differs from "classical" computer-aided design (CAD) in that it is oriented to replace some of the designer's capabilities and not just to support a traditional work-flow with computer graphics and storage capabilities. Nevertheless, CautoD programs most often include $\mathrm{CAD}$ facilities to visualize technical drawings related to the implements of interest. In particular, our LIFTCREATE program is oriented to

\footnotetext{
${ }^{7}$ This distinction is fundamental, because DiSeGnO must know which events have to be associated with input and output web services, respectively.
} 
automating design of elevators, taking the designer from the very first measurements to a complete project which guarantees feasibility within a specific normative framework. LIFTCREATE works in three steps. In the first step, the user is asked to enter relevant parameters characterizing the project, and an overall "design philosophy" to be implemented. For instance, if the size of the elevator's shaft is known and fixed in advance, LIFTCREATE can generate solutions which maximize payload, door size, or car size. A design philosophy is just a set of heuristics which, e.g., prioritize door size over other elements, still keeping into account hard constraints, e.g., payload and car size should not fall below some threshold. In the second phase, LIFTCREATE retrieves components from a database of parts and explores the (combinatorial) space of potential solutions, either using heuristic search techniques, or resorting to optimizations techniques - like those suggested, e.g., in [8]. In the third phase, a set of feasible designs is proposed to the user, sorted according to decreasing relevance considering the initial design philosophy. For instance, if door size is to be maximized, the first alternatives shown to the user are those with the widest doors, perhaps at the expense of payload or car size.

The main issue with LIFTCREATE work-flow is that even simple versions of elevators consists of a large number of components, including car frame, car, doors (car and landing doors), emergency brakes, pistons or cables, motors and control logic. In order to explore the space of potential designs, components cannot be solely available as drawing elements, like in classical CAD solutions, but they must be handled as first class data inside LIFTCREATE logic. This aspect required us to organize the taxonomy related to different kinds of elevators and, for each elevator kind, to structure the components in a part-whole hierarchy. In Figure 6 we show a fragment of the taxonomy for elevators and an example of part-whole structure for a specific elevator kind. In particular, in Figure 6(top), we see that LIFTCREATE classifies Elevator individuals in two main subclasses corresponding to hydraulic-based (HydraulicElevator) and rope-based (RopeElevator) designs. Both subclasses feature additional partitions to handle specific design requirements, e.g., rope elevators can be provided with a reduction gearbox or not, and the drive can be direct of reeved. For one leaf class of the taxonomy, namely OnePistonDirectHydraulicElevator, in Figure 6 (bottom) we show the detailed part-whole diagram, from which we learn that, e.g., the only peculiar aspects of such subclass is to have only one Piston, whereas the remaining components are common to HydraulicElevator or Elevator. Also we can see that the car frame is specific of hydraulic elevators (CarFrameHydra) and it is comprised of several parts, including CarRails, Buffer and Ropes. The relationships encoded in such part-whole hierarchy are instrumental to LIFTCREATE when it comes to handle drawing, storage and retrieval of designs, but also to reason about the various trade-offs of a design when searching in the space of potential solutions.

\section{Conclusions}

Considering the experiences herein outlined, we summarize some lessons learned in applying ontologies for systems engineering. First and foremost, while ontologies provide an effective tool for conceptualizing scenarios as diverse as those considered, some 
ontology-based tools, e.g., DL reasoners, are untenable unless small-to-medium scale systems are considered. In the case of E414 ontology reasoning with an expressive ontology required us to implement strategies to "forget" data to avoid cluttering the reasoner. In the ILS ontology, where SPARQL queries for KPIs are the only reasoning requested and the usage of OWL 2 QL profile banned expressive but hard-to-compute constructs, scaling still requires discarding data using a recency approach. On the other hand, in DiSeGnO and LIFTCREATE, ontologies merely provide means for conceptualizing data and, as such, flexibility is gained without sacrificing performances. The second take-home message is that sublanguages of OWL 2 are adequate for most modeling purposes. With the only exception of E414 ontology, the ones herein considered fit OWL 2 QL constraints which allowed us to combine in a natural way subclassing ("IS-A" relationships) with other kind of object properties (including "HAS-A"). However, the fact that OWL 2 QL ontologies can be compiled to relational databases - as in the case of DiSeGnO - or handled trough an object-persistency module - as in the case of LIFTCREATE - makes their use transparent to other system components. Third, and final point, with the exception of ILS monitoring, none of our applications required the integration of different data sources which is indeed one of the main tasks which ontologies are advocated for. Nevertheless, our experience witnesses that even in single-source data modeling, ontologies provide an excellent mean to bridge the gap between domain experts and computer software designers.

\section{References}

[1] T. Berners Lee, J. Hendler, and O. Lassila. The Semantic Web. The Scientific American Magazine, 2001.

[2] Steffen Staab and Rudi Studer. Handbook on ontologies. Springer Science \& Business Media, 2013.

[3] Cristina De Ambrosi, Cristiano Ghersi, and Armando Tacchella. An ontologybased condition analyzer for fault classification on railway vehicles. In 22 nd Int.l Conference on Industrial, Engineering and Other Applications of Applied Intelligent Systems, IEA/AIE 2009, Tainan, Taiwan, June 24-27, 2009. Proceedings, pages 449-458, 2009.

[4] Matteo Casu, Giuseppe Cicala, and Armando Tacchella. Ontology-based data access: An application to intermodal logistics. Information Systems Frontiers, 15(5):849-871, 2013.

[5] Giuseppe Cicala, Marco De Luca, Marco Oreggia, and Armando Tacchella. A multi-formalism framework to generate diagnostic decision support systems. In 30th European Conference on Modelling and Simulation, ECMS 2016, Regensburg, Germany, May 31 - June 3, 2016, Proceedings., pages 628-634, 2016.

[6] D. Calvanese, G. De Giacomo, D. Lembo, M. Lenzerini, and R. Rosati. DLLite: Tractable Description Logics for Ontologies. In Proceedings of the National 
Conference on Artificial Intelligence, volume 20, page 602. Menlo Park, CA; Cambridge, MA; London; AAAI Press; MIT Press; 1999, 2005.

[7] Johan Eker, Jorn W Janneck, Edward A. Lee, Jie Liu, Xiaojun Liu, Jozsef Ludvig, Sonia Sachs, Yuhong Xiong, and Stephen Neuendorffer. Taming heterogeneity the Ptolemy approach. Proceedings of the IEEE, 91(1):127-144, 2003.

[8] Robin T. Bye, Ottar L. Osen, Birger Skogeng Pedersen, Ibrahim A. Hameed, and Hans Georg Schaathun. A software framework for intelligent computerautomated product design. In 30th European Conference on Modelling and Simulation, ECMS 2016, Regensburg, Germany, May 31 - June 3, 2016, Proceedings., pages 534-543, 2016.

[9] Louis A. Kamentsky and Chao-Ning Liu. Computer-automated design of multifont print recognition logic. IBM Journal of Research and Development, 7(1):213, 1963.

[10] R Venkata Rao and Vimal J Savsani. Mechanical design optimization using advanced optimization techniques. Springer Science \& Business Media, 2012.

[11] E.Sirin, B. Parsia, B. Cuenca Grau, A. Kalyanpur, and Y. Katz. Pellet: A practical owl-dl reasoner. Web Semantics: Science, Services and Agents on the World Wide Web, 5(2):51-53, 2007.

[12] M. Rodriguez-Muro and D. Calvanese. Quest, an OWL 2 QL Reasoner for Ontology-based Data Access. OWLED 2012, 2012.

[13] J.H. Gennari, M.A. Musen, R.W. Fergerson, W.E. Grosso, M. Crubézy, H. Eriksson, N.F. Noy, and S.W. Tu. The Evolution of Protégé: An Environment for Knowledge-Based Systems Development. International Journal of HumanComputer Studies, 58(1):89-123, 2003. 